\title{
Quality of life during olaparib maintenance therapy in platinum-sensitive relapsed serous ovarian cancer
}

Jonathan A Ledermann ${ }^{*}, 1$, Philipp Harter ${ }^{2}$, Charlie Gourley ${ }^{3}$, Michael Friedlander ${ }^{4}$, Ignace Vergote ${ }^{5}$, Gordon Rustin ${ }^{6}$, Clare Scott ${ }^{7}$, Werner Meier ${ }^{8}$, Ronnie Shapira-Frommer ${ }^{9}$, Tamar Safra ${ }^{10}$, Daniela Matei ${ }^{11}$, Anitra Fielding ${ }^{12}$, Bryan Bennett ${ }^{12,14}$, David Parry ${ }^{12}$, Stuart Spencer ${ }^{12}$, Helen Mann ${ }^{12}$ and Ursula Matulonis ${ }^{13}$ ${ }^{1}$ University College London, Cancer Research UK and UCL Cancer Trials Centre, 90 Tottenham Court Road, London W1T 4TJ, UK; ${ }^{2}$ Kliniken Essen Mitte, Henricistraße 95, D-45136 Essen, Germany; ${ }^{3}$ University of Edinburgh Cancer Research UK Centre, MRC IGMM, Western General Hospital, Crewe Road South, Edinburgh EH4 2XR, UK; ${ }^{4}$ UNSW Clinical School, Prince of Wales Hospital, High Street, Randwick, NSW 2031, Australia; ${ }^{5}$ University of Leuven, Herestraat 49, 3000 Leuven, Belgium; ${ }^{6}$ Mount Vernon Hospital, Rickmansworth Road, Northwood HA6 2RN, UK; ${ }^{7}$ Royal Melbourne Hospital, 300 Grattan Street, Parkville, Victoria 3050, Australia; ${ }^{8}$ Evangelisches Krankenhaus, Kirchfeldstraße 40, 40217 Düsseldorf, Germany; ${ }^{9}$ Chaim Sheba Medical Center, Derech Sheba 2, Tel Hashomer 52621, Israel; ${ }^{10}$ Tel Aviv Sourasky Medical Center, 6 Weizmann Street, Tel Aviv 64239, Israel; ${ }^{11}$ Indiana University School of Medicine, 980 West Walnut Street, Indianapolis, IN 46202, USA; ${ }^{12}$ AstraZeneca, Mereside, Alderley Park, Macclesfield SK10 4TG, UK and ${ }^{13}$ Dana-Farber Cancer Institute, 450 Brookline Avenue, Boston, MA 02215, USA

Background: Maintenance monotherapy with the poly(ADP-ribose) polymerase inhibitor olaparib significantly prolongs progression-free survival over placebo in patients with platinum-sensitive relapsed serous ovarian cancer, with greatest benefit seen in patients with a BRCA1/2 mutation (BRCAm). Preservation of health-related quality of life (HRQoL) is important during maintenance therapy; we evaluated the effect of olaparib on HRQoL in this Phase II trial (NCT00753545, Study 19).

Methods: Patients received olaparib $400 \mathrm{mg}$ b.i.d. (capsules) or placebo until progression. Patient-reported HRQoL and diseaserelated symptoms were evaluated using the FACT-Ovarian (FACT-O) questionnaire (completed at baseline and every 28 days until progression), the FACT/NCCN Ovarian Symptom Index (FOSI) and the Trial Outcome Index (TOI). TOI of the FACT-O was the primary measure.

Results: Overall, 265 women were randomised to maintenance olaparib $(n=136)$ or placebo $(n=129)$. Compliance for HRQoL assessment was high ( $80 \%$ over time). Most patients in both arms reported a best response of 'no change' on TOI (81\%) and other HRQoL measures. There were no statistically significant differences in time to worsening or improvement rates of TOI, FOSI and FACT-O scores in the overall, BRCAm and germline BRCAm populations.

Conclusions: Maintenance treatment with olaparib was well tolerated and had no adverse impact on HRQoL in this study of patients with platinum-sensitive relapsed serous ovarian cancer who had responded to their most recent platinum-based therapy (partial or complete response). Interpretation of the HRQoL results in this population may differ from patients who have not responded to their most recent platinum-based therapy.

${ }^{*}$ Correspondence: Professor JA Ledermann; E-mail: j.ledermann@ucl.ac.uk
${ }^{14}$ Current address: Adelphi Values, Adelphi Mill, Grimshaw Lane, Bollington SK10 5JB, UK

Received 9 February 2016; revised 23 September 2016; accepted 30 September 2016; published online 8 November 2016 (c) 2016 Cancer Research UK. All rights reserved 0007-0920/16 
Health-related quality of life (HRQoL) is a multidimensional concept encompassing: physical, cognitive and emotional wellbeing; social functioning domains; disease-related symptoms; therapy-induced side effects; and potential financial and family burden. These HRQoL measures are a particularly important consideration in the maintenance setting after response to chemotherapy, when the majority of patients do not have any symptoms related to recurrent cancer, as the aim of maintenance treatment is to prolong the time to progression and to delay the need for further chemotherapy without compromising the quality of life of the patients on treatment (Friedlander and King, 2013).

Olaparib (Lynparza) is an oral poly(ADP-ribose) polymerase (PARP) inhibitor that blocks base-excision repair by trapping PARP at sites of DNA damage, leading to synthetic lethality in tumour cells with deficiencies in homologous recombination repair, such as those with $B R C A 1 / 2$ mutations (BRCAm) (Evers et al, 2008; Rottenberg et al, 2008). Olaparib has been extensively studied and in several Phase II trials, olaparib monotherapy exhibited antitumour activity in patients with breast and ovarian cancer, particularly in those with BRCAm (Audeh et al, 2010; Tutt et al, 2010; Gelmon et al, 2011). In December 2014, olaparib obtained regulatory approval in the EU as maintenance monotherapy for adult patients with platinum-sensitive recurrent (PSR) $B R C A m$ (germline and/or somatic) high-grade serous ovarian cancer (SOC), fallopian tube or primary peritoneal cancer, who are in complete or partial response to platinum-based chemotherapy.

This EU approval was based on the results of a randomised, double-blind, placebo-controlled, Phase II study (NCT00753545, D0810C00019, Study 19), in which maintenance monotherapy with olaparib (capsules) significantly prolonged progression-free survival (PFS) vs placebo in patients with PSR SOC and patients with a BRCAm were most likely to benefit from treatment (Ledermann et al, 2012, 2014). The toxicity profile from this study demonstrated that adverse events (AEs) were manageable in most patients (Ledermann et al, 2012, 2014). Dose reductions were performed as a result of AEs in $24 \%$ and $4 \%$ of patients in the olaparib and placebo arms, respectively. The common AEs of nausea, vomiting, fatigue and anaemia led to dose reduction in $4 \%$, $3 \%, 4 \%$ and $4 \%$ of olaparib patients $(0 \%, 1 \%, 1 \%$ and $1 \%$ of placebo patients), respectively. The discontinuation rate as a result of AEs was $4.4 \%$ for olaparib patients and $1.6 \%$ for placebo patients. HRQoL was assessed as a secondary objective in this study and we report the impact of olaparib on HRQoL and disease-related symptoms.

\section{MATERIALS AND METHODS}

Study design and patients. In this Phase II trial (NCT00753545), adult patients with platinum-sensitive, relapsed, high-grade SOC who had received at least two platinum-based regimens and had a partial or complete response to their most recent platinum-based regimen were enrolled (Ledermann et al, 2012). Patients were randomised to receive olaparib $400 \mathrm{mg}$ capsules or placebo twice daily within 8 weeks of completing platinum-based chemotherapy. Assessment of BRCAm status was not required at enrolment and was either reported on case report forms after local testing or established retrospectively using blood samples (germline BRCAm $(\mathrm{g} B R C A \mathrm{~m}))$ and/or archival tumour samples (tumour $B R C A \mathrm{~m}$ ) (Ledermann et al, 2014). The trial design, including inclusion/ exclusion criteria, and a planned retrospective analysis of outcomes by $B R C A$ status have been published previously (Ledermann et al, $2012,2014)$. The secondary endpoints reported here had the same data cut-off as the primary analysis (30 June 2010). All patients provided written informed consent. The institutional review boards or independent ethics committees of all investigational sites approved the protocol. The study was performed in accordance with the Declaration of Helsinki, Good Clinical Practice and the AstraZeneca Policy on Bioethics (AstraZeneca, 2015).

HRQoL tool selection. HRQoL analysis assessed the impact of maintenance therapy with olaparib, relative to placebo, on HRQoL and disease-related symptoms in patients with PSR SOC. HRQoL was assessed using the Functional Assessment of Cancer Therapy Ovarian (FACT-O) questionnaire, which is a multidimensional questionnaire developed and validated for ovarian cancer patients. The FACT-O questionnaire was linguistically validated in multiple languages; however, no Ukrainian translation was available.

Assessments. The FACT-O questionnaire comprises questions regarding physical, social, emotional and functional wellbeing and additional concerns $(n=7,7,6,7$ and 12 items, respectively; Supplementary Material and Supplementary Figure S1).

The Trial Outcome Index (TOI), a subset analysis of the FACT-O, was the primary HRQoL measure. Whereas the FACT-O score derives from 39 items (score range: $0-152$; higher score indicates better health state), the TOI score derives from 26 physical and functional wellbeing items and ovarian cancer concerns subscales (score range: $0-104$ ).

The Functional Assessment of Cancer Therapy/National Comprehensive Cancer Network Ovarian Symptom Index (FOSI) assessment derives from eight symptom-related FACT-O items (score range $0-32$ ). Individual concepts of nausea, vomiting and fatigue were assessed from specific FACT-O questions ('I have nausea', 'I have been vomiting', 'I have a lack of energy') that were also captured in FOSI scores (Supplementary Material).

There is currently no standard HRQoL assessment for use in oncology maintenance clinical trials; the TOI of the FACT-O was chosen as the primary HRQoL assessment in this study because it is a validated tool and contained the most concepts of importance to ovarian cancer patients and those that a pharmacological product would be expected to impact (physical well-being, functional well-being and specific concerns for ovarian cancer patients). The FACT-O is a multidimensional questionnaire developed and validated for use by ovarian cancer patients; it includes the 27-item FACT-General (FACT-G) targeted to general cancer patients and 12 questions specific to issues faced by ovarian cancer patients (FACT-O subscale). The FACT-G questionnaire includes the following four subscales: physical well-being (PWB; seven items), social well-being (seven items), emotional well-being (six items) and functional well-being (FWB; seven items). These subscales can be analyzed separately or aggregated to produce a total HRQoL score. The FACT-G has demonstrated reliability, validity and responsiveness to change over time (Cella et al, 1993). Two of the FACT-G subscales (PWB and FWB) plus the FACT-O subscale are summed to represent the TOI.

Analyses. Patients completed the FACT-O questionnaire at baseline and monthly until progression. If patients discontinued for reasons other than progression (assessed by Response Evaluation Criteria in Solid Tumours (RECIST)), HRQoL assessments continued until progression was confirmed. Individual symptom severity over the previous 7 days was measured using the five-item Likert scale (not at all (0), a little bit (1), somewhat (2), quite a bit (3) and very much (4)). Better wellbeing was generally indicated by higher scores; where appropriate, raw scores were reversed (Supplementary Material).

Improvement and worsening rates in HRQoL values were evaluated against prospectively determined minimally important differences relevant to each endpoint (Supplementary Material) (Osoba et al, 2005).

A retrospective exploratory analysis using linear mixed-model repeated-measures (MMRM) modelling, adjusting for score at baseline, time and treatment-by-time interaction, estimated the 
mean effect over time for HRQoL (Stockler et al, 2014). Estimates of the least-squares means for treatment effects within and between treatment groups were reported with corresponding 95\% confidence intervals (CIs). Mixed-model repeated-measures analyses were performed on the overall population, as well as the $B R C A m$ and gBRCAm subgroups.

\section{RESULTS}

Patients. Baseline demographics have been reported for the overall population and BRCAm subgroups (Ledermann et al, 2012, 2014) and are summarised, alongside data for $\mathrm{gBRCAm}$ patients, in Table 1. No significant differences in patient demographics were observed between treatment groups. Following retrospective $\mathrm{g} B R C A \mathrm{~m}$ and somatic $B R C A \mathrm{~m}$ testing, $B R C A \mathrm{~m}$ status data were available for 254 of 265 patients (96\%), of whom 136 of 254 (54\%) had a known/suspected deleterious gBRCAm and/or somatic BRCAm (Ledermann et al, 2014). Ninety-six patients (36\%) had a gBRCAm (Ledermann et al, 2014).

For the 265 randomised patients ( $n=136$ olaparib, $n=129$ placebo), compliance with study treatment was good (mean $97 \%$ (standard deviation (s.d.) 9\%) olaparib, mean 99\% (s.d. 3\%) placebo). Compliance rates for TOI, FOSI and FACT-O assessment were high at baseline and similar in each arm $(85 \%, 86 \%$ and $84 \%$ for olaparib-treated patients $v s 86 \%, 89 \%$ and $86 \%$ for placebotreated patients, respectively). Overall compliance rates over 16 months were also high $(69 \%, 70 \%$ and $69 \%$ for olaparib-treated patients and $69 \%, 70 \%$ and $69 \%$ for placebo-treated patients, respectively). Compliance rates over time for the first 6 months of treatment for TOI, FACT-O and FOSI are detailed in Supplementary Table S1. A total of 41 patients were excluded from HRQoL analyses ( $15 \% ; n=23$ olaparib, $n=18$ placebo); 33 (12\%; $n=19$ olaparib, $n=14$ placebo) did not complete the FACT-O questionnaire at baseline or were not evaluable for FACT-O/TOI and were excluded from HRQoL analyses. Of these patients, 14 required a Ukrainian translation $(n=9$ olaparib, $n=5$ placebo), which was unavailable, 20 did not receive the questionnaire because of administrative failure $(n=11$ olaparib, $n=9$ placebo), two patients (olaparib) were not evaluable for FACT-O and five patients were not evaluable for FACT-O and TOI ( $n=1$ olaparib, $n=4$ placebo). Patient-level HRQoL data were collected until either progression or the primary analysis. As the median PFS from randomisation after the end of chemotherapy was 4.8 months in the placebo arm, the majority of placebo patients $(68 \%)$ did not contribute HRQoL data beyond 6 months. Therefore, no data are presented beyond 6 months.

HRQoL. At baseline mean (standard deviation) scores were balanced between olaparib- and placebo-treated patients for all assessments (TOI, FOSI and FACT-O; Table 2). Across the overall, $B R C A \mathrm{~m}$ and gBRCAm groups, most patients achieved best HRQoL responses of 'no change' (Table 2). Results from MMRM analyses involving $B R C A \mathrm{~m}$ or $\mathrm{gBRCAm}$ patients were consistent with results from patients irrespective of $B R C A \mathrm{~m}$ status.

TOI. Most patients reported a best response of 'no change' (Table 2); statistical analysis of improvement rates showed no statistically significant difference between treatment groups in the overall population (odds ratio (OR) 1.14; 95\% CI 0.58, 2.24; $P=0.7)$. For $B R C A m$ and $\mathrm{gBRCAm}$ patients, there was no statistically significant difference in improvement rates, although numerically more patients receiving olaparib had a best response of 'improved' vs placebo (BRCAm: $25 \%$ vs 19\%, respectively; OR 1.37; 95\% CI 0.56, 3.46; $P=0.5$; gBRCAm: $27 \%$ vs $8 \%$, respectively; OR 4.08; 95\% CI 1.11, 19.9; $P=0.03$ ), indicating no detriment to HRQoL. Supplementary Table S2 provides further details for the time-to-worsening analysis using Cox's proportional hazards model for TOI.

Patients had high baseline TOI scores (mean \pm s.d., $81.7 \pm 11.8$ with olaparib and $81.5 \pm 11.6$ with placebo in the overall population; $79.9 \pm 12.1$ and $79.5 \pm 12.1$, respectively, for $B R C A m$ patients; $79.5 \pm 12.3$ and $81.0 \pm 11.0$, respectively, for $\mathrm{gBRCAm}$ patients), with TOI scores remaining consistent over time and similar between groups (Figure $1 \mathrm{a}-\mathrm{c}$ and Supplementary Figure

Table 1. Patient baseline characteristics among all patients randomised in the Phase II study (NCT00753545) (Ledermann et al, 2012, 2014)

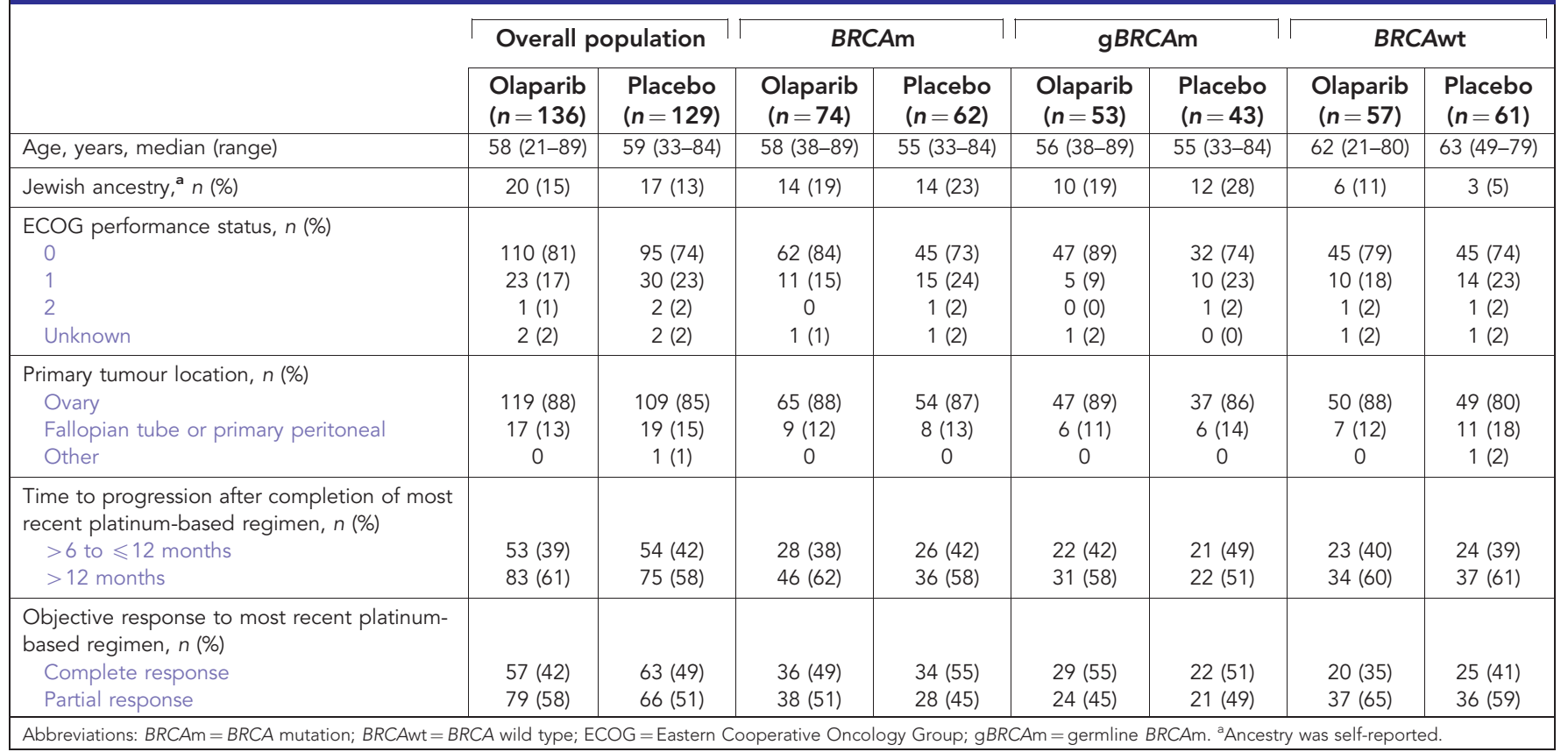


Table 2. HRQoL best response for the overall population and by BRCA status

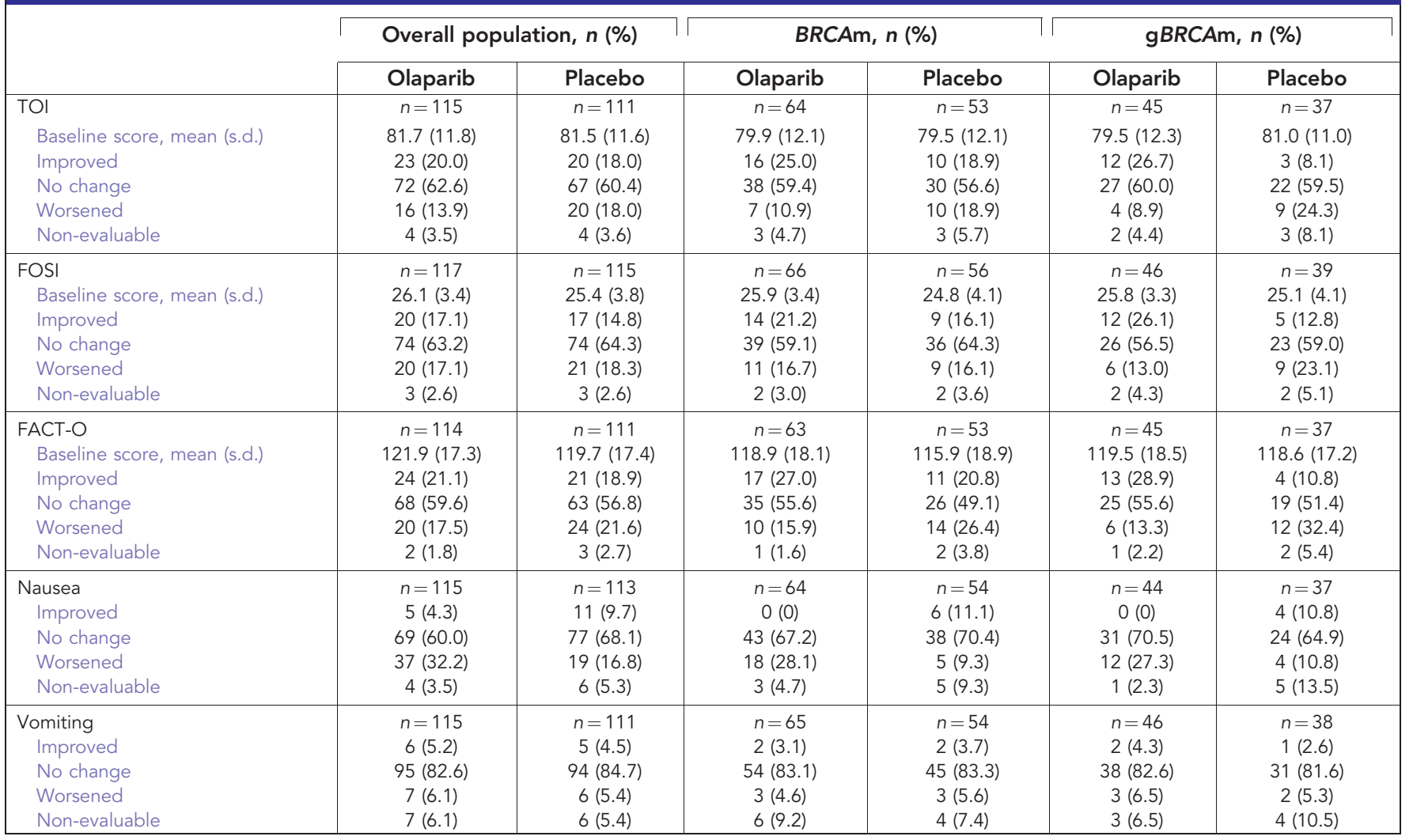

S2a and b). Details of further subscales are provided in Supplementary Figure S3. The greatest decrease was observed at month 1 for olaparib and month 4 for placebo (overall population, $B R C A \mathrm{~m}$ and $\mathrm{gBRCAm}$ ). In the overall population, there was no statistically significant difference in median time to TOI worsening with olaparib $v s$ placebo, although the median time to worsening was numerically shorter with olaparib (3.8 vs 4.6 months, respectively; hazard ratio (HR) $1.08 ; 95 \%$ CI $0.75,1.55 ; P=0.7$ ). Median time to TOI worsening was numerically longer with olaparib vs placebo for $B R C A \mathrm{~m}$ patients (5.7 vs 3.7 months, respectively; HR $0.8 ; 95 \% \mathrm{CI} 0.48,1.34 ; P=0.4)$ and $\mathrm{gBRCAm}$ patients (7.4 vs 3.6 months, respectively; HR 0.54 95\% CI 0.30 , 0.99; $P=0.048)$. There was no HRQoL detriment in the overall, $B R C A \mathrm{~m}$ and $\mathrm{gBRCAm}$ populations.

FOSI. In the overall population, the percentage of patients with a best response of 'improved' in FOSI was similar between treatment groups ( $17 \%$ vs $15 \%$ for olaparib vs placebo; Table 2 ), with no significant differences (OR 1.22;95\% CI 0.60, 2.51; $P=0.59$ ). These values were $21 \%$ vs $16 \%$, respectively, in BRCAm patients (OR $1.41 ; 95 \%$ CI $0.56,3.70 ; P=0.47$ ) and $26 \%$ vs $13 \%$, respectively, in gBRCAm patients (OR 2.31; 95\% CI 0.75, 8.10; $P=0.15)$. Supplementary Table S2 provides further details for the time-to-worsening analysis using Cox's proportional hazards model for FOSI.

Patient experience of important symptoms on FOSI remained consistent with baseline and comparable between treatment groups and over time (Figure $2 \mathrm{a}-\mathrm{c}$ and Supplementary Figure S2c-d). Baseline mean FOSI scores \pm s.d. were $26.1 \pm 3.4$ with olaparib and $25.4 \pm 3.8$ with placebo for the overall population; $25.9 \pm 3.4$ and $24.8 \pm 4.1$, respectively, for $B R C A m$ patients; and $25.8 \pm 3.3$ and $25.1 \pm 4.1$, respectively, for $\mathrm{g} B R C A \mathrm{~m}$ patients. In the overall population, median time to FOSI worsening was $2.8 v s 3.7$ months for olaparib and placebo, respectively; there was no significant difference (HR 1.22; 95\% CI 0.88, $1.71 ; P=0.228$ ). Median time to FOSI worsening was 2.8 vs 3.7 months, respectively, in BRCAm patients (HR 1.15; 95\% CI 0.74, 1.81; $P=0.53$ ) and 3.7 vs 3.3 months, respectively, in gBRCAm patients (HR $0.71 ; 95 \%$ CI 0.42 , $1.22 ; P=0.212$ ).

FACT-O. No statistically significant differences were observed between treatment groups ( $21 \%$ vs $19 \%$ for olaparib vs placebo) for improvement in the FACT-O score in the overall population (OR $1.17 ; 95 \%$ CI $0.60,2.27 ; P=0.65)$. In BRCAm patients, the percentage of patients with a best response of 'improved' was $27 \%$ vs $21 \%$ for olaparib vs placebo, respectively (OR 1.38; 95\% CI 0.58 , $3.39 ; P=0.47)$. These values were $29 \%$ vs $11 \%$, respectively, for gBRCAm patients (OR 3.26; 95\% CI 1.00, 12.9; $P=0.05$ ). Supplementary Table S2 provides further details for the time-toworsening analysis using Cox's proportional hazards model for FACT-O.

The FACT-O total score remained consistent with baseline and comparable between groups and over time (Figure $3 \mathrm{a}-\mathrm{c}$ and Supplementary Figure S2e-f). Baseline mean FACT-O scores \pm s.d. were $121.9 \pm 17.3$ with olaparib and $119.7 \pm 17.4$ with placebo for the overall population; $118.9 \pm 18.1$ and $115.9 \pm 18.9$, respectively, for BRCAm patients; and $119.5 \pm 18.5$ and $118.6 \pm 17.2$, respectively, for $\mathrm{g} B R C A \mathrm{~m}$ patients. Time to FACT-O score worsening in the overall population was numerically shorter with olaparib vs placebo (2.8 vs 4.6 months, respectively; HR 1.16; 95\% CI 0.83 , $1.64 ; P=0.39$ ). There were no clinically relevant or statistically significant differences in FACT-O score time to worsening for BRCAm patients (3.2 vs 4.4 months, respectively; HR 1.04; $95 \%$ CI $0.65,1.69 ; P=0.87$ ) or $\mathrm{gBRCAm}$ patients (3.2 vs 3.7 months, respectively; HR 0.84; 95\% CI 0.48, 1.48; $P=0.55)$.

Nausea, vomiting and fatigue. Minimal changes were observed during the course of treatment for nausea, vomiting and fatigue 
in the overall population (Supplementary Figure S4). Most patients did not report nausea at baseline or discontinuation. At baseline, the mean nausea score was 3.77 with olaparib and 3.71 with placebo in the overall population, with a shorter median time to nausea worsening with olaparib (1.1 months) than placebo (6.5 months). Although olaparib patients experienced more nausea during the first months of treatment and a higher proportion had a best response of 'worsening' vs placebo $(32.2 \%$ vs $16.8 \%$, respectively), scores became similar with increasing time on olaparib. In gBRCAm patients, decreases in nausea scores were transient and only observed at month 1 . The percentage of patients with a score of 3 or 4 (symptoms: 'a little bit' or 'not at all') for nausea was consistently above $65 \%$ (olaparib) and $75 \%$ (placebo) in the overall population;
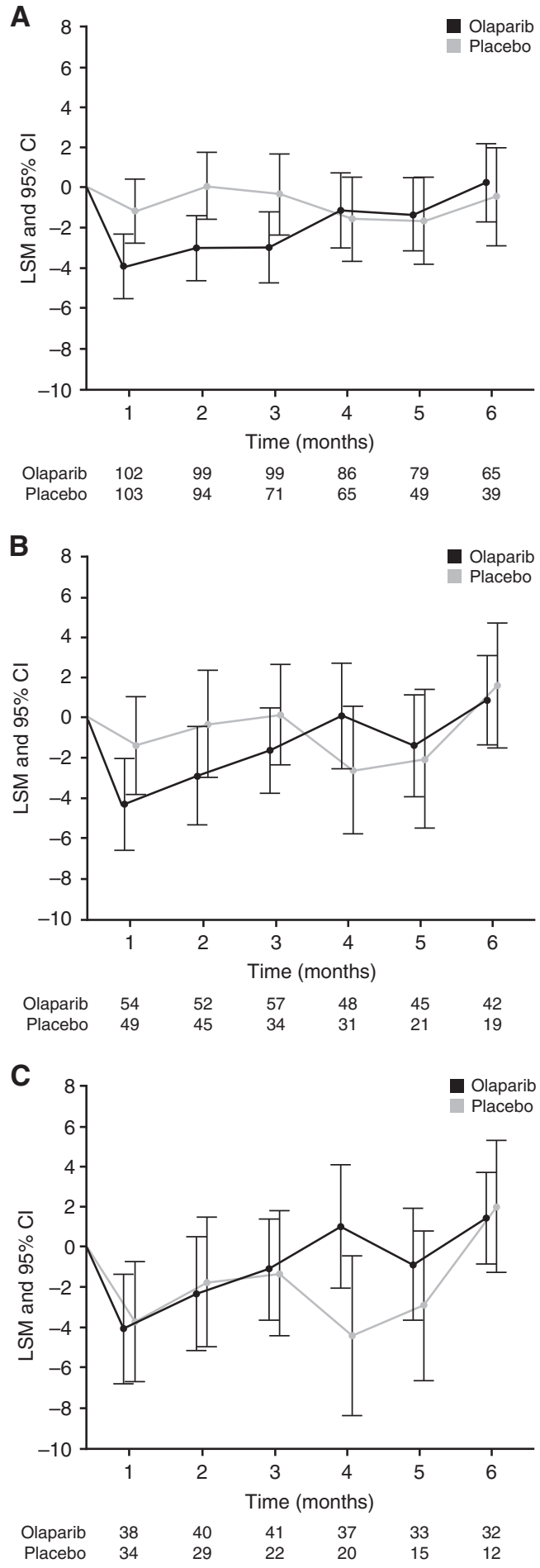

Figure 1. TOI change from baseline to 6 months for (A) the overall population and patients with (B) BRCAm and (C) gBRCAm. Worsening of TOI indicated with a negative score. Olaparib/Placebo indicates the number of patients who completed the assessment at each time point. Abbreviations: $\mathrm{Cl}=$ confidence interval; $\mathrm{LSM}=$ least squares mean.
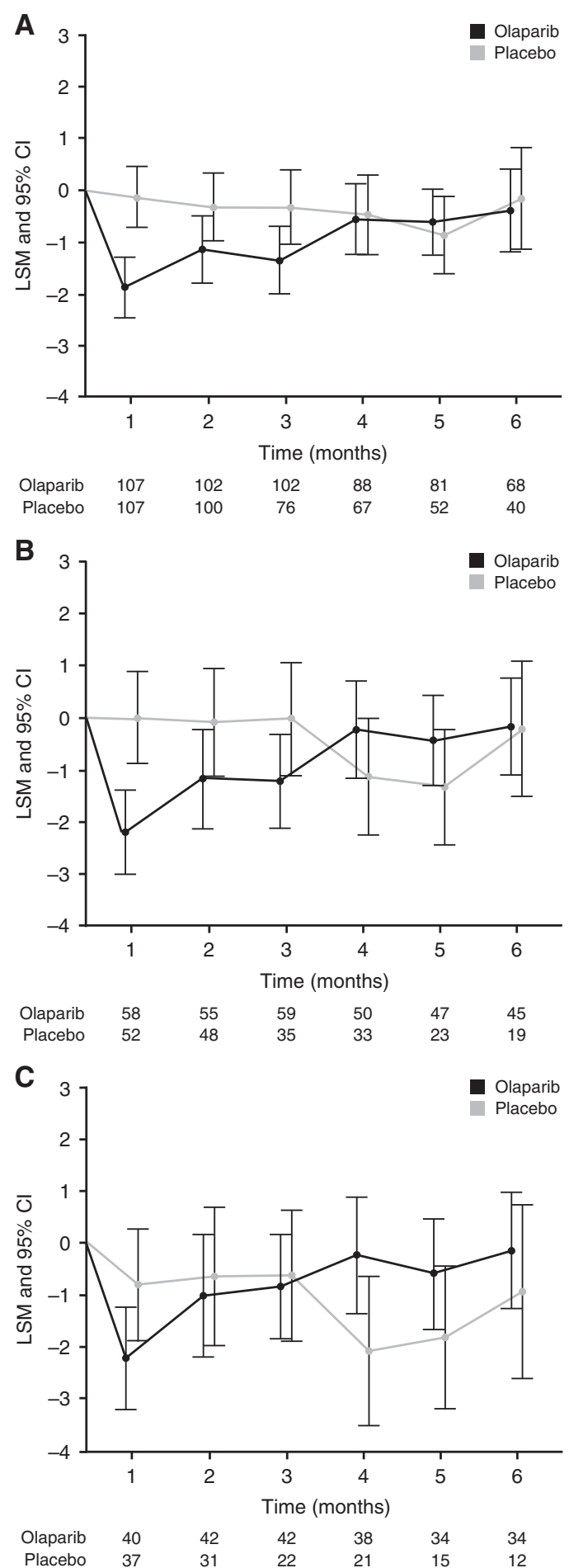

Figure 2. FOSI change from baseline to 6 months for (A) the overall population and patients with (B) BRCAm and (C) gBRCAm. Worsening of FOSI indicated with a negative score. Olaparib/Placebo indicates the number of patients who completed the assessment at each time point. Abbreviations: $\mathrm{Cl}=$ confidence interval; $\mathrm{LSM}=$ least squares mean. 
these values were 60 and $65 \%$, respectively, for $B R C A m$ patients and $60 \%$ and $75 \%$, respectively, for $\mathrm{g} B R C A \mathrm{~m}$ patients. There were no differences in vomiting or fatigue scores between the
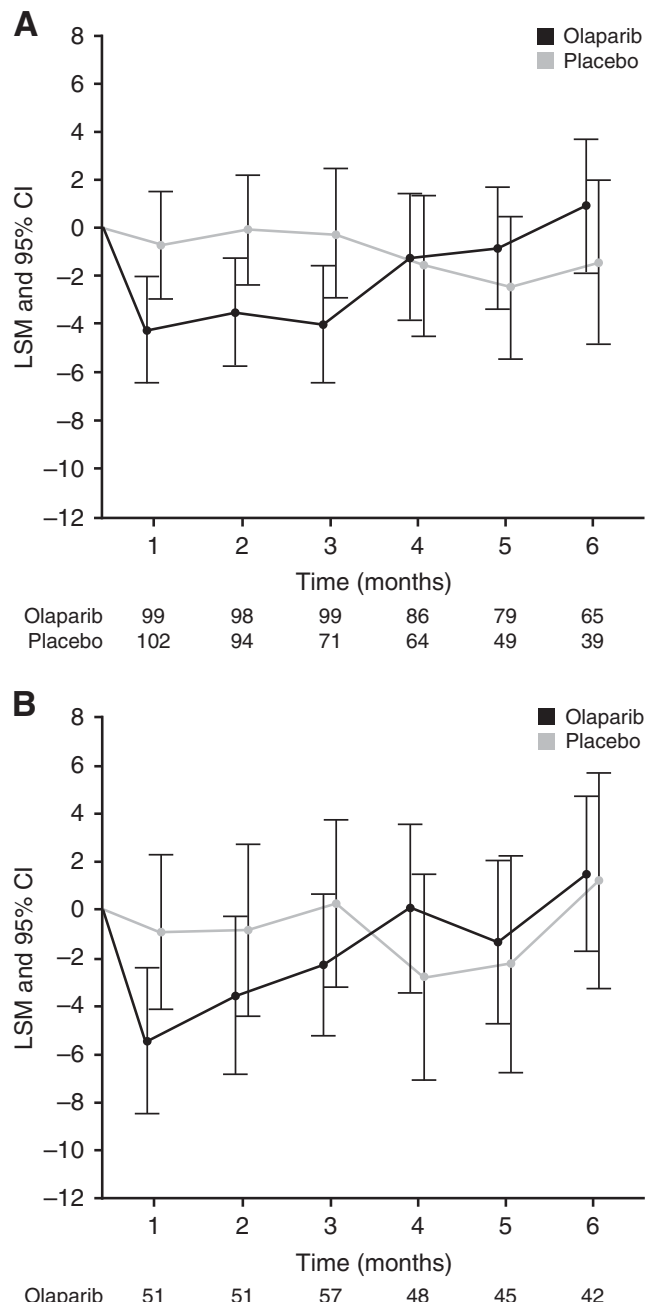

$\begin{array}{lllllll}\text { Olaparib } & 51 & 51 & 57 & 48 & 45 & 42 \\ \text { Placebo } & 48 & 45 & 34 & 30 & 21 & 19\end{array}$

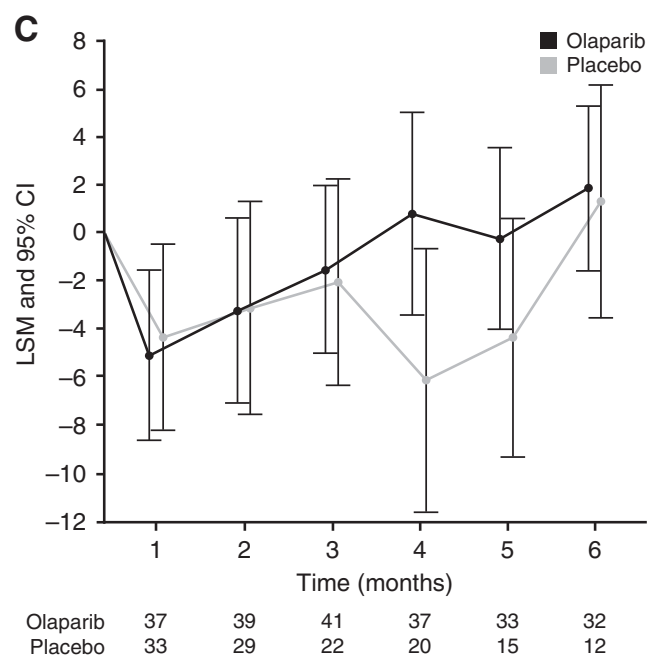

Figure 3. FACT-O total score change from baseline to 6 months for (A) the overall population and patients with (B) BRCAm and (C) gBRCAm. Worsening of FACT-O indicated with a negative score. Olaparib/Placebo indicates the number of patients who completed the assessment at each time point. Abbreviations: $\mathrm{Cl}=$ confidence interval; $\mathrm{LSM}=$ least squares mean. olaparib and placebo groups in the overall, $B R C A \mathrm{~m}$ or $\mathrm{g} B R C A \mathrm{~m}$ populations (Supplementary Figure S4b and c).

\section{DISCUSSION}

Stability of HRQoL with acceptable side effects of treatment is an important consideration for patients with recurrent ovarian cancer receiving maintenance therapy after response to chemotherapy. In this study, there were no statistically significant or clinically relevant differences in HRQoL between treatment arms on TOI, total FACT-O and FOSI assessments among all patients and in the $B R C A \mathrm{~m}$ or gBRCAm subgroups. These data demonstrate that maintenance treatment with olaparib had no apparent adverse impact on HRQoL in patients with PSR SOC, which is also supported by the high compliance with treatment and very low discontinuation rates because of AEs.

Evidence of maintained HRQoL complements and provides additional support of the primary study results of a significantly prolonged PFS with olaparib vs placebo (difference in median PFS 3.6 months, $P<0.001$ ), with $B R C A \mathrm{~m}$ patients most likely to benefit (difference in median PFS 6.9 months, $P<0.0001$ ). In addition, AEs in this study were generally manageable (Ledermann et al, 2012 , 2014). Olaparib did not appear to affect patients' experience of vomiting, with similar scores between olaparib- and placebotreated patients across all time points. Olaparib patients experienced more early nausea and, to a lesser extent, fatigue, but this is consistent with known $\mathrm{AE}$ profiles and, with increasing time on therapy, scores became similar to those for placebo. This is reflected by the initial worsening of physical wellbeing scores in olaparib-treated patients, however, after 4 months of treatment with olaparib the HRQoL scores were similar to those in patients treated with placebo.

The patient population evaluated in the current study included patients with complete or partial responses to chemotherapy. As expected, baseline HRQoL scores in this group were relatively high compared with scores from other cancer cohorts. These high baseline scores are a consideration when interpreting time-toworsening measures, particularly those for vomiting, which was not a prominent symptom impacting patients in either arm of the study at entry. A trend towards a higher proportion of patients in the overall population reporting improvements in TOI, FOSI and total FACT-O following treatment with olaparib $v s$ placebo was seen across the endpoints evaluated. It is possible that any improvement during the maintenance treatment phase reflects recovery from the side effects of prior treatment, rather than from amelioration of cancer-related symptoms, as these would be expected to be minimal at study entry for patients whose disease is in response.

The HRQoL finding in gBRCAm patients is worth emphasising. Although the least number of patients was included in this subgroup analysis, patient numbers were not much smaller than those in the BRCAm subset. As the study design did not include a spending strategy to control type I error rates among the patientreported outcome (PRO) endpoints, all analyses should be considered exploratory and the $P$-values nominal. Of the 136 $B R C A m$ patients in this Phase II study, 96 were known to have a gBRCAm (Ledermann et al, 2014). It is unknown whether these findings originate from a biological basis.

Limitations of this study include HRQoL data not being collected beyond disease progression. Patients with progressive disease usually receive further courses of chemotherapy, an intervention associated with reductions in HRQoL. Olaparib significantly delays progression (Ledermann et al, 2012); therefore, post-progression patient outcome data may have indicated a benefit with olaparib. Delays in time to first subsequent therapy or 
death (TFST) may also delay detriments in HRQoL; it is of interest that future studies collect HRQoL data during PFS, TFST and beyond. Additionally, this analysis is limited in that primary and secondary PRO hypotheses were not defined prior to the study. At the time of the development of this study, there were limited studies on the QoL of cancer patients receiving maintenance treatment and there were no specifically agreed PRO or QoL assessments for use in this setting in oncology trials. Studies in other cancer types, such as non-small-cell lung cancer and metastatic breast cancer, where maintenance treatment is more common, have used a range of QoL assessments, including cancerspecific variations of the EORTC QLQ-C30, EuroQol 5-dimensional questionnaire (EQ-5D) and FACT tools (Gridelli et al, 2012; Park et al, 2015; Spigel et al, 2015). More recently, the importance of QoL assessments during clinical trials has been noted (Friedlander and King, 2013) and specific guidance such as the CONSORT-PRO recommendations should be followed in future trials (Calvert et al, 2013). The Phase III study of olaparib maintenance treatment in patients with BRCAm PSR ovarian cancer (SOLO2; NCT01874353) has included several predefined QoL endpoints to better assess the impact of this treatment on QoL. Finally, although the compliance rate of approximately $80 \%$ is favourable, it is incomplete. Reasons for reduced HRQoL assessment completion included no validated Ukrainian translation of the FACT-O questionnaire and administrative failure in questionnaire distribution.

In conclusion, olaparib maintenance therapy demonstrated no detrimental impact on HRQoL outcomes compared with placebo. Phase III trials of olaparib in patients with $B R C A$-mutated ovarian cancer are ongoing (NCT01844986, NCT01874353 and NCT02282020). These studies will assess HRQoL using the FACT-O and EQ-5D-5L (five-level EuroQol) assessments and will collect HRQoL data over a longer period, beyond disease progression.

\section{ACKNOWLEDGEMENTS}

Medical writing assistance during the development of this manuscript was provided by Rachel Patel from Mudskipper Business Ltd, funded by AstraZeneca. This work was supported by funding from AstraZeneca.

\section{CONFLICT OF INTEREST}

JAL has participated in an advisory board for AstraZeneca. PH has acted in an advisory role for AstraZeneca and received honoraria from AstraZeneca. CG has acted in an advisory role for AstraZeneca, Clovis, Roche and Nucana, received honoraria from AstraZeneca and Roche, and received research funding from AstraZeneca, Novartis and Aprea. MF has participated in advisory boards for AstraZeneca, Clovis, Pfizer and Roche and received honoraria from AstraZeneca, Pfizer and Roche. IV has acted in an advisory role for AstraZeneca. GR has participated in advisory boards for AstraZeneca and Clovis. CS has acted in an advisory role for AstraZeneca, Clovis and Eisai Australia, received honoraria from AstraZeneca and Roche, provided expert testimony on behalf of AstraZeneca and received remuneration for travel from AstraZeneca. RS-F has participated in an advisory board for AstraZeneca. TS has acted in an advisory role for AstraZeneca and Clovis and received honoraria from AstraZeneca and Clovis. DM has acted in an advisory role for AstraZeneca and received honoraria from AstraZeneca. AF, DP, SS and HM are employees of AstraZeneca and own stock. UM has acted in an advisory role for
AstraZeneca and Tesaro. All remaining authors declare no conflicts of interest.

\section{REFERENCES}

AstraZeneca (2015) Global Policy: Bioethics. Available at https:// www.astrazeneca.com/sustainability/responsible-research.html.

Audeh MW, Carmichael J, Penson RT, Friedlander M, Powell B, Bell-McGuinn KM, Scott C, Weitzel JN, Oaknin A, Loman N, Lu K, Schmutzler RK, Matulonis U, Wickens M, Tutt A (2010) Oral poly (ADP-ribose) polymerase inhibitor olaparib in patients with $B R C A 1$ or $B R C A 2$ mutations and recurrent ovarian cancer: a proof-of-concept trial. Lancet 376: 245-251.

Calvert M, Blazeby J, Altman DG, Revicki DA, Moher D, Brundage MD (2013) Reporting of patient-reported outcomes in randomized trials: the CONSORT PRO extension. JAMA 309: 814-822.

Cella DF, Tulsky DS, Gray G, Sarafian B, Linn E, Bonomi A, Silberman M, Yellen SB, Winicour P, Brannon J (1993) The Functional Assessment of Cancer Therapy scale: development and validation of the general measure. J Clin Oncol 11: 570-579.

Evers B, Drost R, Schut E, de Bruin M, van der Burg E, Derksen PW, Holstege H, Liu X, van Drunen E, Beverloo HB, Smith GC, Martin NM, Lau A, O'Connor MJ, Jonkers J (2008) Selective inhibition of BRCA2-deficient mammary tumor cell growth by AZD2281 and cisplatin. Clin Cancer Res 14: 3916-3925.

Friedlander ML, King MT (2013) Patient-reported outcomes in ovarian cancer clinical trials. Ann Oncol 24(Suppl 10): x64-x68.

Gelmon KA, Tischkowitz M, Mackay H, Swenerton K, Robidoux A, Tonkin K, Hirte H, Huntsman D, Clemons M, Gilks B, Yerushalmi R, MacPherson E, Carmichael J, Oza A (2011) Olaparib in patients with recurrent high-grade serous or poorly differentiated ovarian carcinoma or triple-negative breast cancer: a phase 2, multicentre, open-label, non-randomised study. Lancet Oncol 12: 852-861.

Gridelli C, De Marinis F, Pujol JL, Reck M, Ramlau R, Parente B, Pieters T, Middleton G, Corral J, Winfree K, Melemed S, Zimmermann A, John W, Beyrer J, Chouaki N, Visseren-Grul C, Paz-Ares LG (2012) Safety, resource use, and quality of life in paramount: a phase III study of maintenance pemetrexed versus placebo after induction pemetrexed plus cisplatin for advanced nonsquamous non-small-cell lung cancer. J Thorac Oncol 7: 1713-1721.

Ledermann J, Harter P, Gourley C, Friedlander M, Vergote I, Rustin G, Scott C, Meier W, Shapira Frommer R, Safra T, Matei D, MacPherson E, Watkins C, Carmichael J, Matulonis U (2012) Olaparib maintenance therapy in platinum-sensitive relapsed ovarian cancer. N Engl J Med 366: 1382-1392.

Ledermann J, Harter P, Gourley C, Friedlander M, Vergote I, Rustin G, Scott CL, Meier W, Shapira-Frommer R, Safra T, Matei D, Fielding A, Spencer S, Dougherty B, Orr M, Hodgson D, Barrett JC, Matulonis U (2014) Olaparib maintenance therapy in patients with platinum-sensitive relapsed serous ovarian cancer: a preplanned retrospective analysis of outcomes by BRCA status in a randomised phase 2 trial. Lancet Oncol 15 : 852-861.

Osoba D, Bezjak A, Brundage M, Zee B, Tu D, Pater J (2005) Analysis and interpretation of health-related quality-of-life data from clinical trials: basic approach of The National Cancer Institute of Canada Clinical Trials Group. Eur J Cancer 41: 280-287.

Park YH, Jung KH, Im SA, Sohn JH, Ro J, Ahn JH, Kim SB, Nam BH, Oh dY, Han SW, Lee S, Park IH, Lee KS, Kim JH, Kang SY, Lee MH, Park HS, Woo SY, Jung SH, Ahn JS, Im YH (2015) Quality of life (QoL) in metastatic breast cancer patients with maintenance paclitaxel plus gemcitabine (PG) chemotherapy: results from phase III, multicenter, randomized trial of maintenance chemotherapy versus observation (KCSG-BR07-02). Breast Cancer Res Treat 152: 77-85.

Rottenberg S, Jaspers JE, Kersbergen A, van der Burg E, Nygren AO, Zander SA, Derksen PW, de Bruin M, Zevenhoven J, Lau A, Boulter R, Cranston A, O'Connor MJ, Martin NM, Borst P, Jonkers J (2008) High sensitivity of BRCA1deficient mammary tumors to the PARP inhibitor AZD2281 alone and in combination with platinum drugs. Proc Natl Acad Sci USA 105: 17079-17084. Spigel DR, Patel JD, Reynolds CH, Garon EB, Hermann RC, Govindan R, Olsen MR, Winfree KB, Chen J, Liu J, Guba SC, Socinski MA, Bonomi P (2015) Quality of life analyses from the randomized, open-label, phase III PointBreak study of 
pemetrexed-carboplatin-bevacizumab followed by maintenance pemetrexedbevacizumab versus paclitaxel-carboplatin-bevacizumab followed by maintenance bevacizumab in patients with stage IIIB or IV nonsquamous nonsmall-cell lung cancer. J Thorac Oncol 10: 353-359.

Stockler MR, Hilpert F, Friedlander M, King MT, Wenzel L, Lee CK, Joly F, de GN, Arranz JA, Mirza MR, Sorio R, Freudensprung U, Sneller V, Hales G, PujadeLauraine E (2014) Patient-reported outcome results from the open-label phase III AURELIA trial evaluating bevacizumab-containing therapy for platinumresistant ovarian cancer. J Clin Oncol 32: 1309-1316.

Tutt A, Robson M, Garber JE, Domchek SM, Audeh MW, Weitzel JN, Friedlander M, Arun B, Loman N, Schmutzler RK, Wardley A, Mitchell G,
Earl H, Wickens M, Carmichael J (2010) Oral poly(ADP-ribose) polymerase inhibitor olaparib in patients with $B R C A 1$ or $B R C A 2$ mutations and advanced breast cancer: a proof-of-concept trial. Lancet 376: $235-244$.

This work is published under the standard license to publish agreement. After 12 months the work will become freely available and the license terms will switch to a Creative Commons AttributionNonCommercial-Share Alike 4.0 Unported License.

Supplementary Information accompanies this paper on British Journal of Cancer website (http://www.nature.com/bjc) 